\title{
INVESTIGATIONS INTO LOAD SPECTRA OF UAVS AIRCRAFT
}

\author{
Mirosław Rodzewicz \\ Dominik Glowacki
}

\author{
Warsaw University of Technology \\ Institute of Aeronautics and Applied Mechanics \\ Warsaw, Poland
}

\begin{abstract}
The paper contains a description of a novel approach to the load spectra estimation applied to UAVs. The authors have developed a number of tools in the LabVIEW environment enabling an indepth analysis of flight-log data. One major achievement was the separation of the load spectra induced by steering and the load spectra induced by turbulence. The authors have shown a significant influence of both of the main load sources on the fatigue life of the UAV airframe, calculated based on the P-M hypothesis. This approach to fatigue testing of composite UAV airframes needs to take into account the rate of load variations as these may affect the fatigue life of tested structures. The paper presents several schemes of calculation algorithms and a number of well-illustrated examples of the tests and investigations results.
\end{abstract}

Keywords: UAV loads, load spectrum, manual control, autopilot.

\section{INTRODUCTION}

The rapid development of Unmanned Aerial Vehicles (UAVs) has been observed since the 1990s, when the usefulness of these aircraft was confirmed during military operations. This development is a result of rapid technological advancement (including information technologies) (Fig.1).

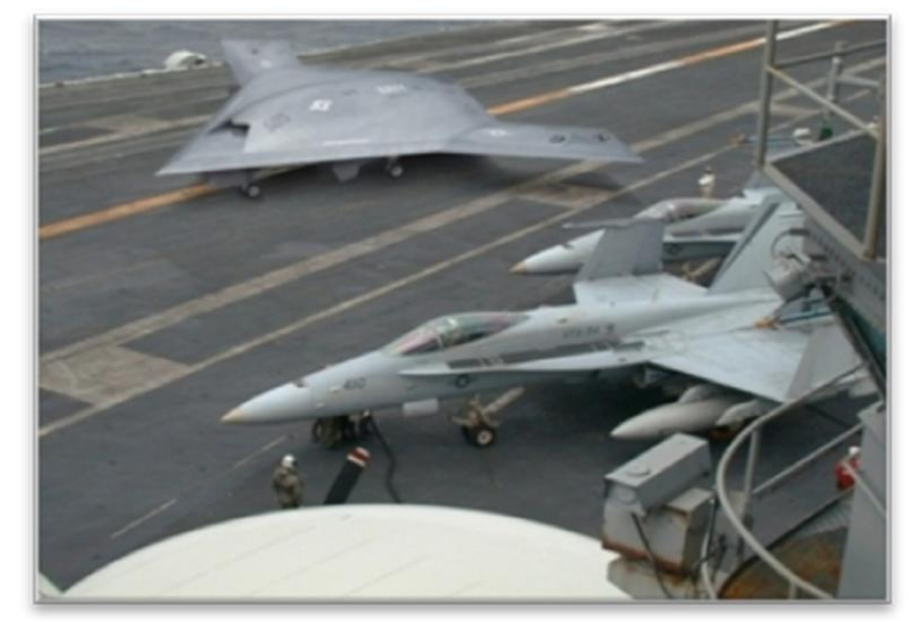

Fig 1. Military $U A V-X-37$

The recent years have seen the development of UAVs for civilian purposes. Users can be found in different fields, e.g. among land surveying companies, meteorological institutions, universities, 
government departments, etc. Potential applications include monitoring of borders, observations during disasters, monitoring of forests, plants and animals, road and railway communications, etc. The construction and entry to service of new aircraft must be preceded by studies on safety during operation.

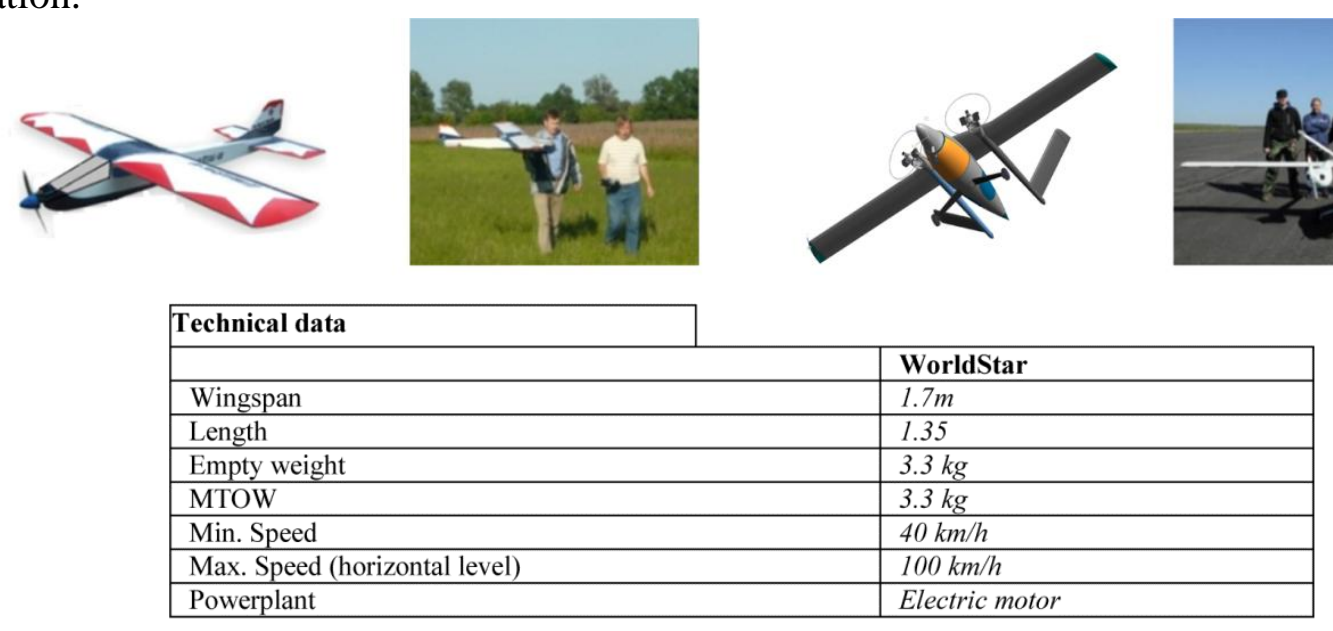

Fig. 2. UAVs made at the WUT and their technical data

A major problem involved in the implementation of a new aircraft is to determine its safe service life. In practice, there are two ways of determining the life of an aircraft. The first method is based on operating experience gained during the use of similar types of UAVs. Since it is just an estimation, this method is less reliable. The second method is based on fatigue tests, during which anticipated loads are applied to the aircraft. In order to estimate the fatigue life either by calculation or in an experimental way, the airframe load spectra must be known (Fig.3).

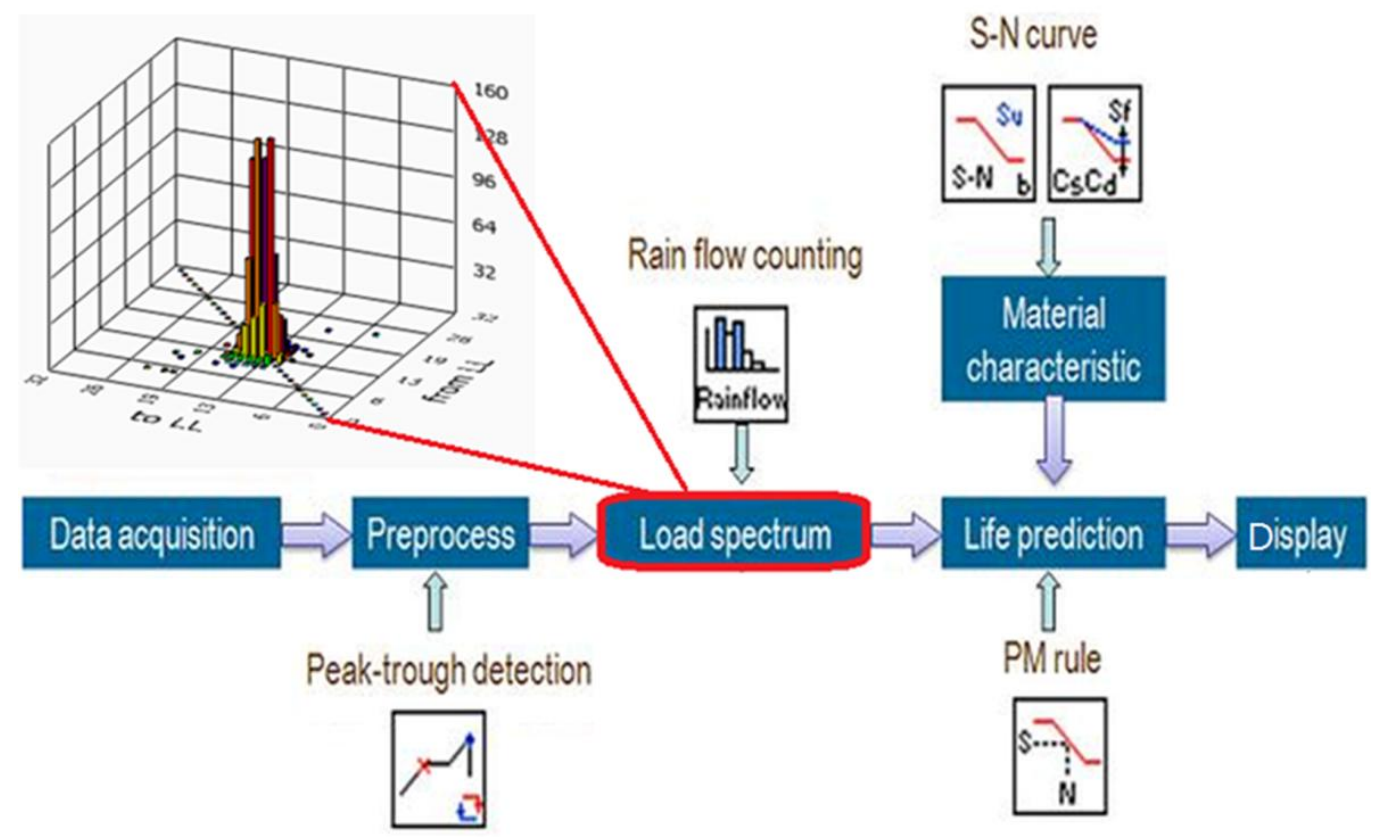

Fig. 3. Calculating schema of fatigue life

The LabVIEW software (Laboratory Virtual Instrument Engineering Workbench) - developed by the National Instruments was used for all the analysis conducted. LabVIEW uses a graphical programming language called "G". This software is often used by research centers to perform tests during which the measurements are made and collected, and the data are analysed. The code 
consists of connected icons, and the execution of the program determines the flow of data between the icons. Some screenshots of the programs for visualization and data processing developed in LabVIEW are shown in Figure 4.
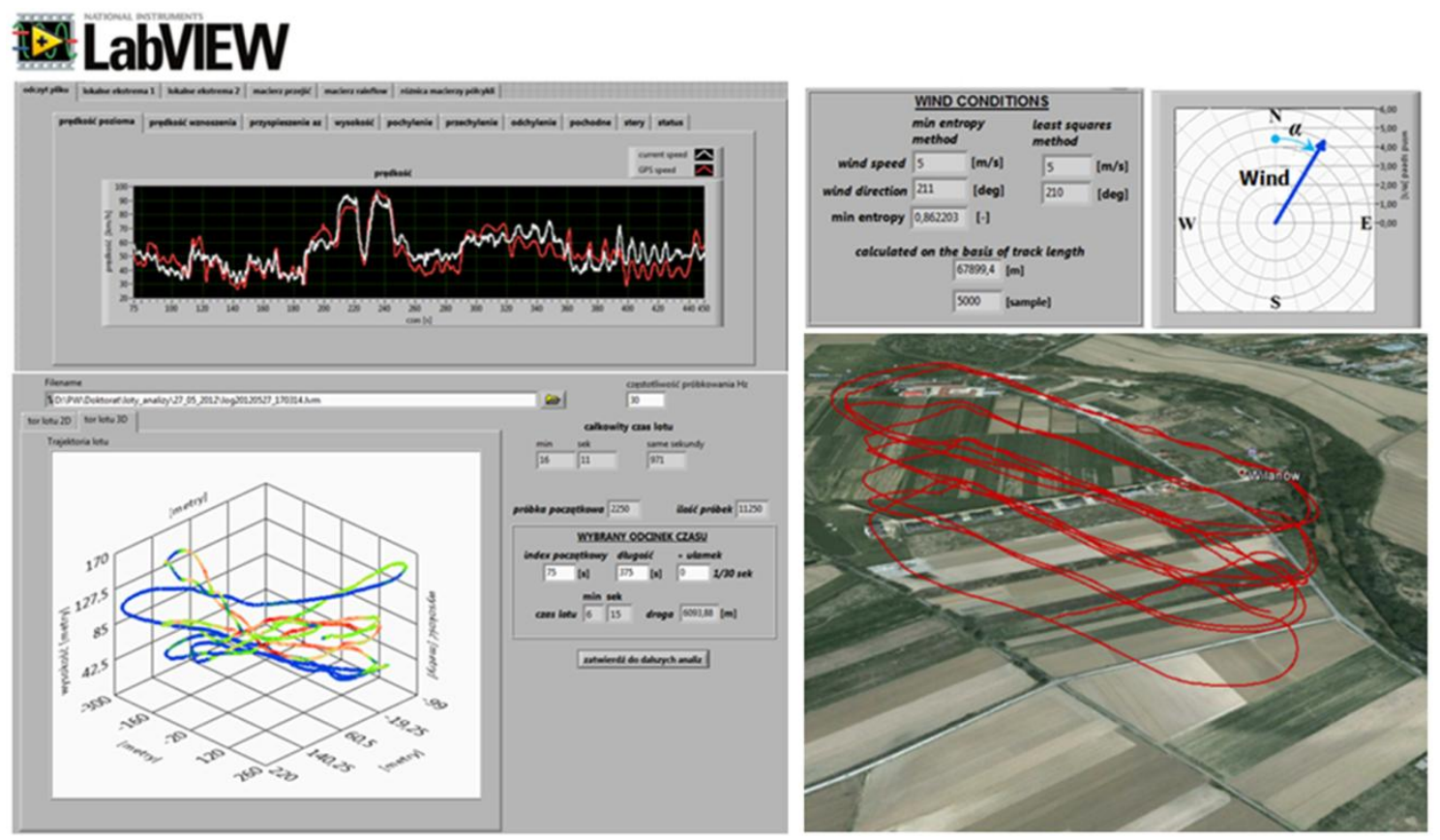

Fig. 4. Examples of screenshots from program developed to analyze UAV's loads in the LabVIEW environment

LabVIEW enables to present 3-D images of the flight trajectory where the colour indicates airspeed or the load factor. Another possibility is the graphical presentation of the steering (control) modes used during the whole flight [1].
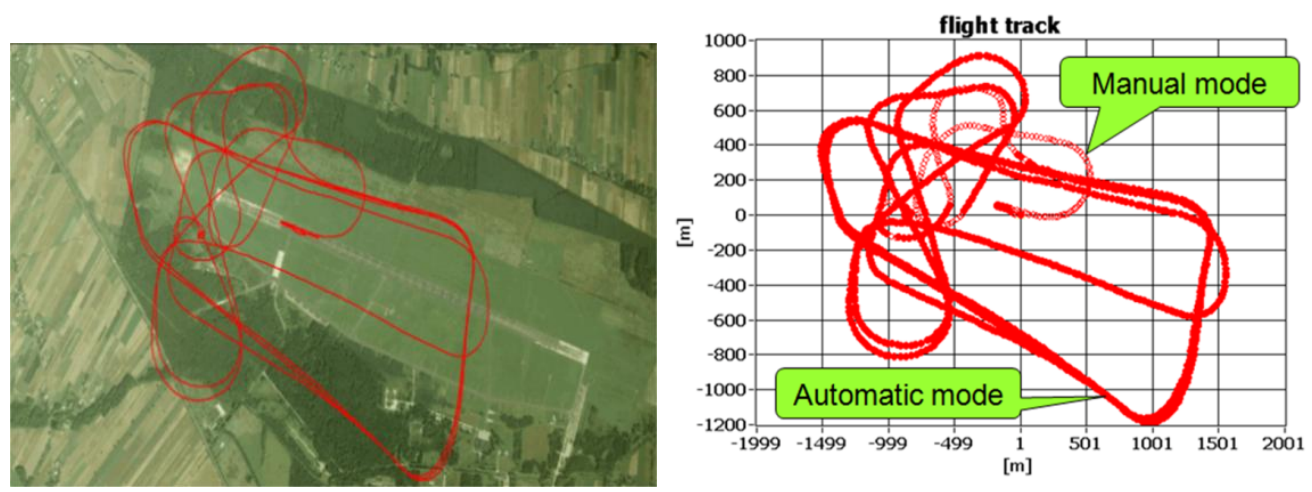

Fig. 5. Visualization of the flight path on Google maps (left) and indication of control modes (right)

The LabView algorithms designed at the WUT allow not only for analyzing and presenting data directly recorded in the log, but also for deriving additional useful information such as wind speed and gust distribution measured during the flight.

\section{HALF-CYCLES ARRAY AS A TOOL FOR LOAD-SIGNAL ANALYSIS}

The authors have adopted the German system of standardization used for gliders, motor gliders and light aircraft where the range of operating loads $\mathbf{n}_{\mathbf{z}}$ is from -3 to 6 (Fig. 6). The measuring 
range was divided into 64 levels (referred to as Load Levels) with the values $\boldsymbol{n}_{z}=-3$ corresponding to the level $\mathrm{LL}=62$, and $\boldsymbol{n}_{z}=6 \rightarrow \mathrm{LL}=6$. Levels $\mathrm{LL}=1,2,3,4,5$ and $\mathrm{LL}=63,64$ are treated as a reserve in case the loads occurring during the flight exceed the permissible load. The scaling equation takes the following form:

$L L=-6.222 n_{z}+43.333$

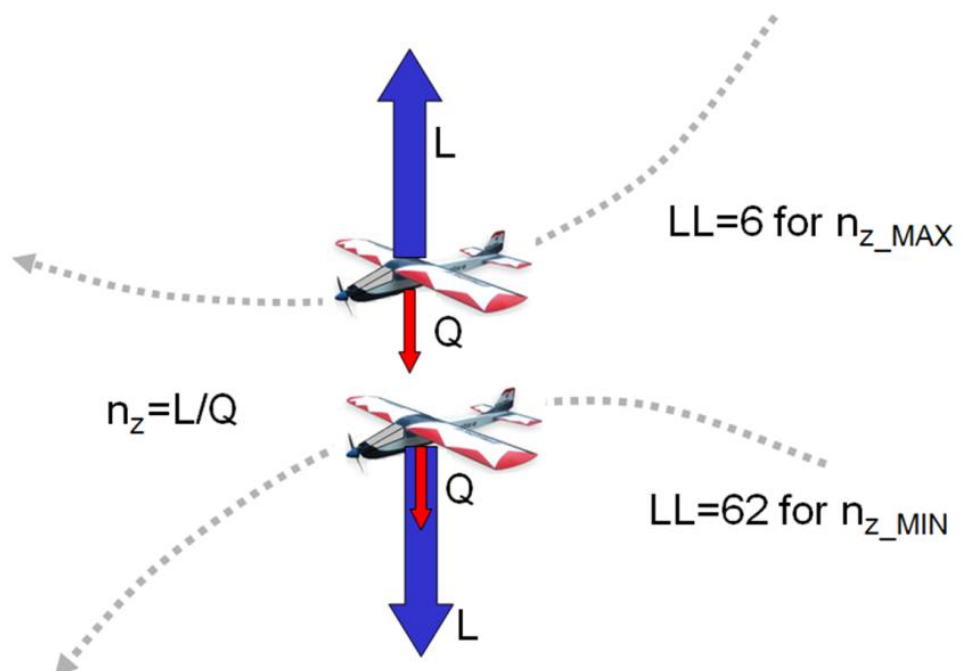

Fig. 6. Correlation between Load Levels and the load factor

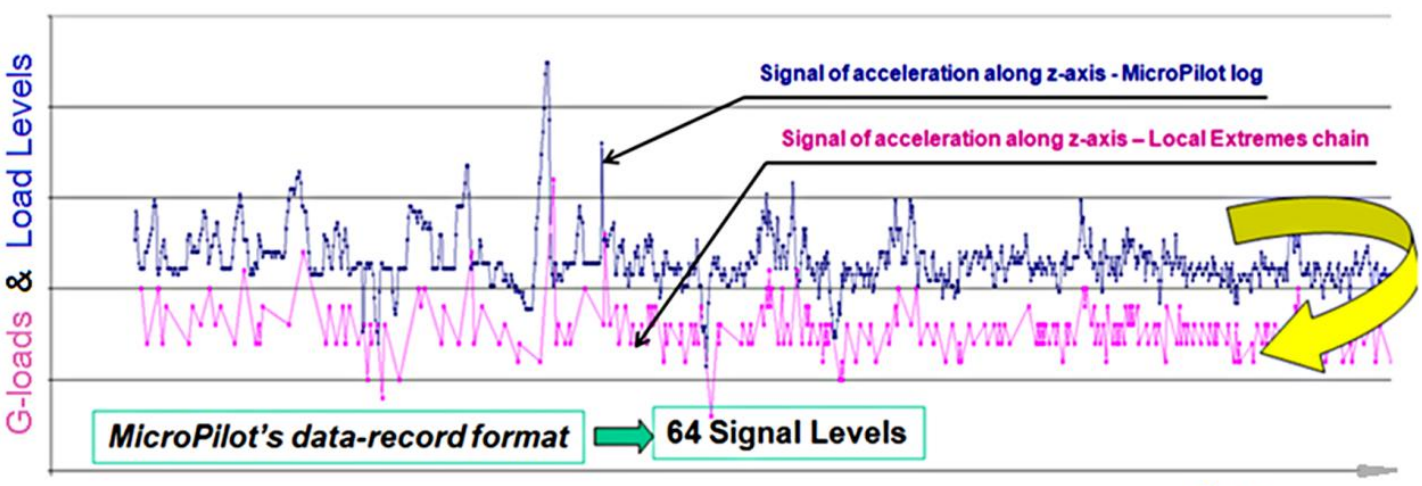

time

Fig. 7. Comparison of the row signal of a g-acceleration and the signal reduced to the chain of local extremes

Load spectrum can be presented as a half-cycle array (HC-array). Creating such an array is associated with the use of the "Rainflow counting algorithm" developed in 1969 by Matsuishi and Endo as one of the most effective methods used for determining full load cycles (i.e. load increments resulting from several load changes). The name "half-cycle array" comes from the assumed rule that each single load cycle consists of two half-cycles that are displayed simultaneously over and under the diagonal-line of the HC-array.

A pre-processed signal in the form of local extremes (Fig. 7) is being analyzed. Figure 8 shows the algorithm for deriving the local extremes vector. This algorithm also allows tracking the speed of load variations. As a result,3 separate vectors of "local extremes," "time vector", and "velocity vector" were obtained (Fig. 8). 


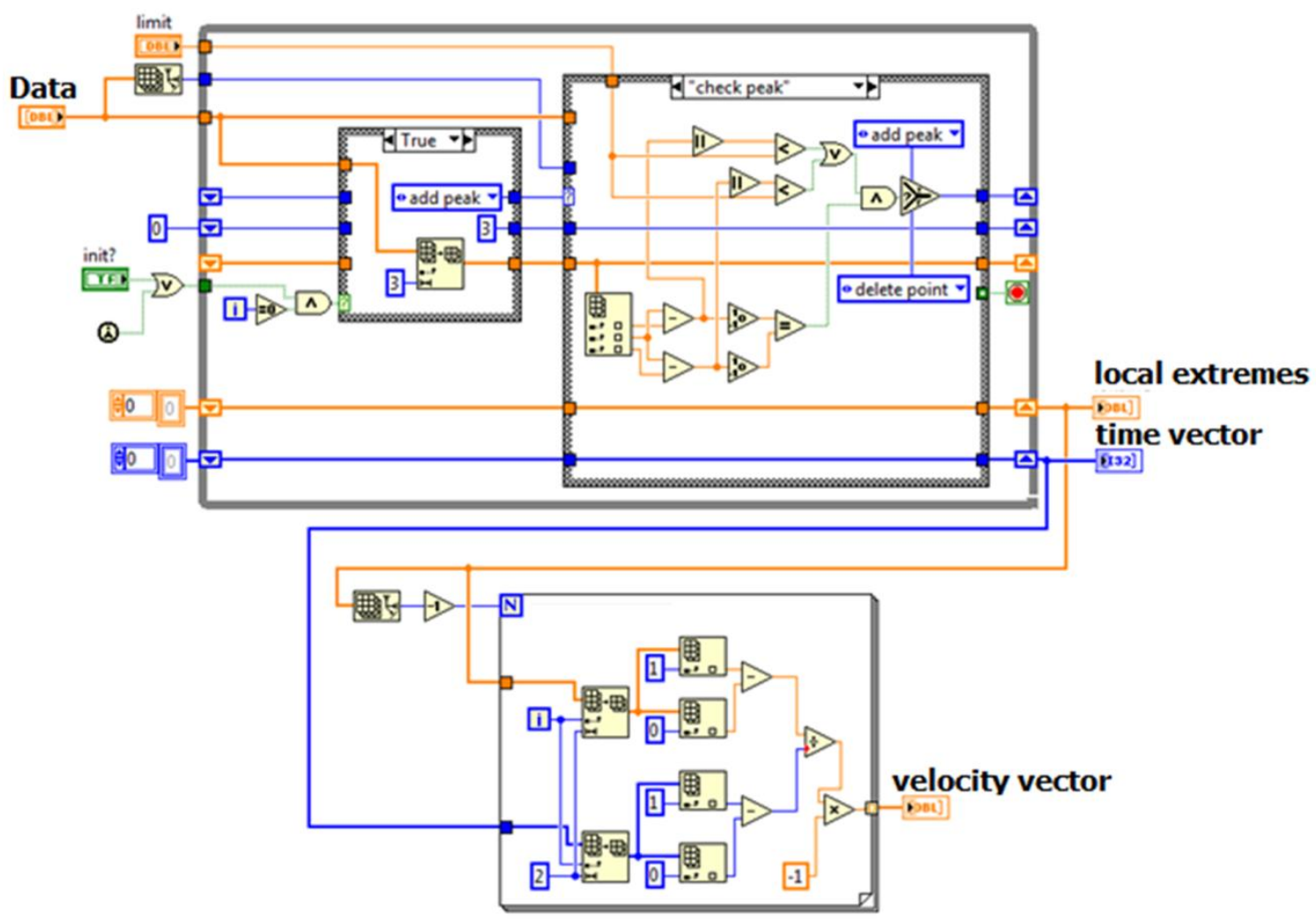

Fig. 8. Algorithm for preparing a chain of load signal local extremes associated with a time vector

The idea of the Rainflow counting algorithm is based on the analogy between the load halfcycles and the "water trickles" when the local extremes vector is positioned vertically (with the time vector directed downwards), and is treated as a multi-stage "pagoda" roof exposed to rain. To derive the half-cycle array it is necessary to count all "trickles" on both sides of the load local extremes graph and to insert them into appropriate cells of the array. This procedure is described in $[2,3,4]$.

The schema of HC-array derivation based on the Rainflow counting algorithm is shown in Figure 9.

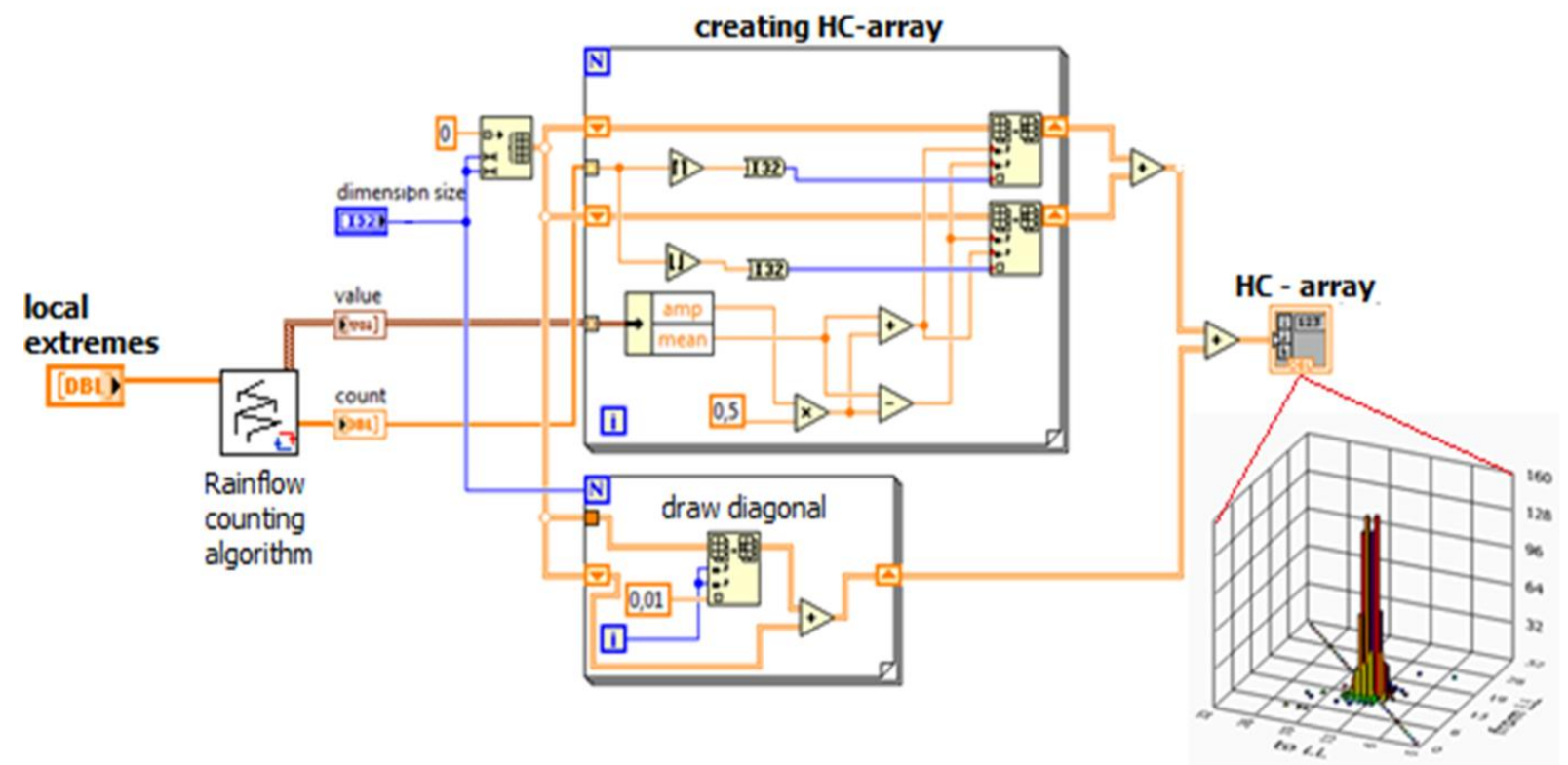

Fig. 9. Half-cycle array derivation algorithm 
Very convenient for comparison of load spectra is the so-called incremental load spectrum (ILS). It represents graphically the number of occurrences for each load level. The ILS is formed from the summation of the values of the cells over and under the strip made along the main diagonal of the array as shown in Figure 10. An increase in the value of the load by at least one level is equal to the sum of all the values of the cells; an increase in the value of the load by at least two levels is equal to the sum of all table cells without first cells adjacent to the diagonal. In order to calculate the number of occurrences for the next load levels, it is necessary to move further away from the main diagonal of the array.

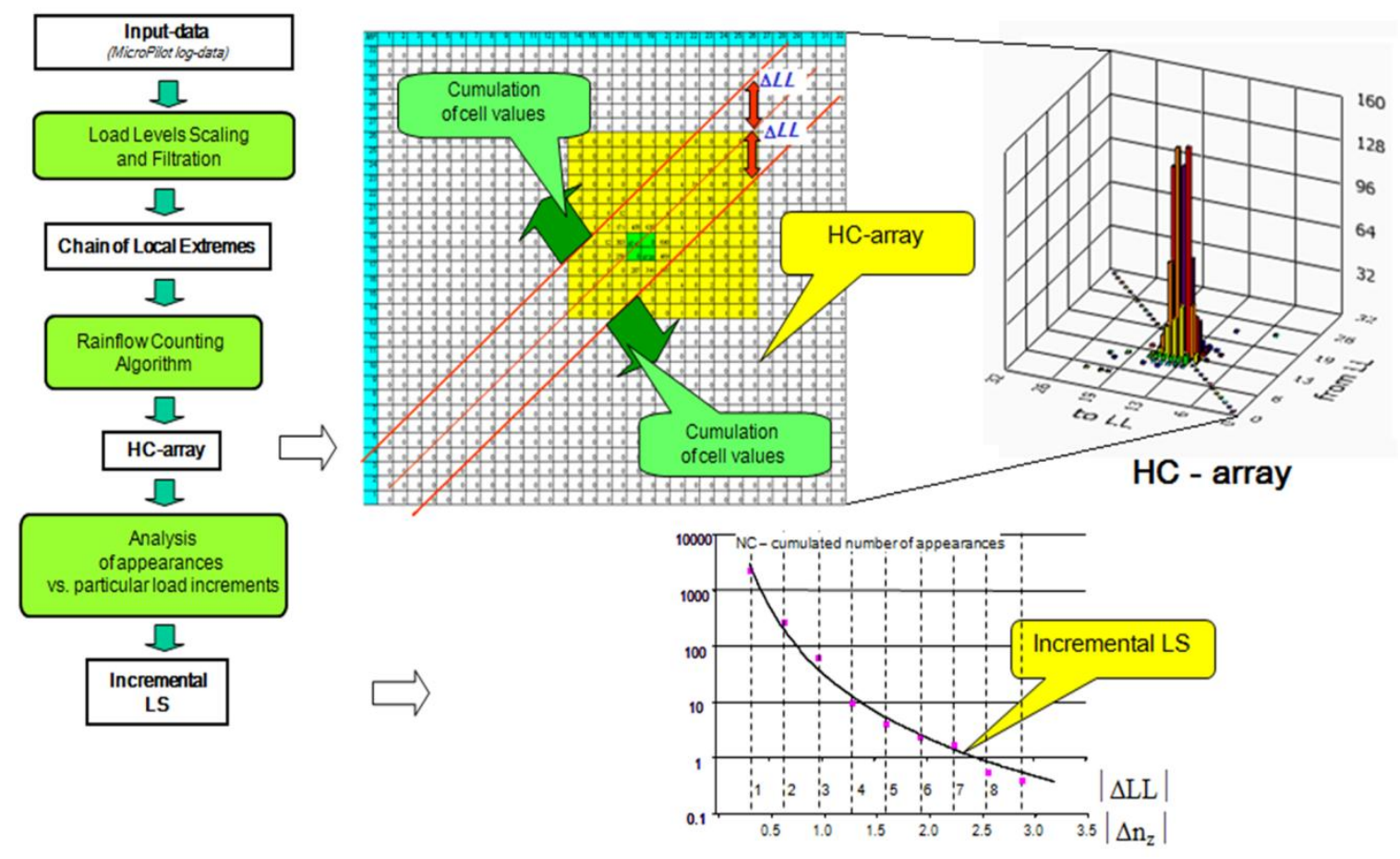

Fig. 10. Derivation of Incremental Load Spectrum

\section{LOAD SPECTRA FOR THE UAV CONTROLLED IN A MANUAL OR AUTOMATIC WAY IN VARIOUS WEATHER CONDITIONS}

The tools described in the previous section were used to test the loads of UAVs in different both weather conditions and operating modes. The qualitative nature of the loads in the manual and automatic control mode is shown in Figures 11 and 12.
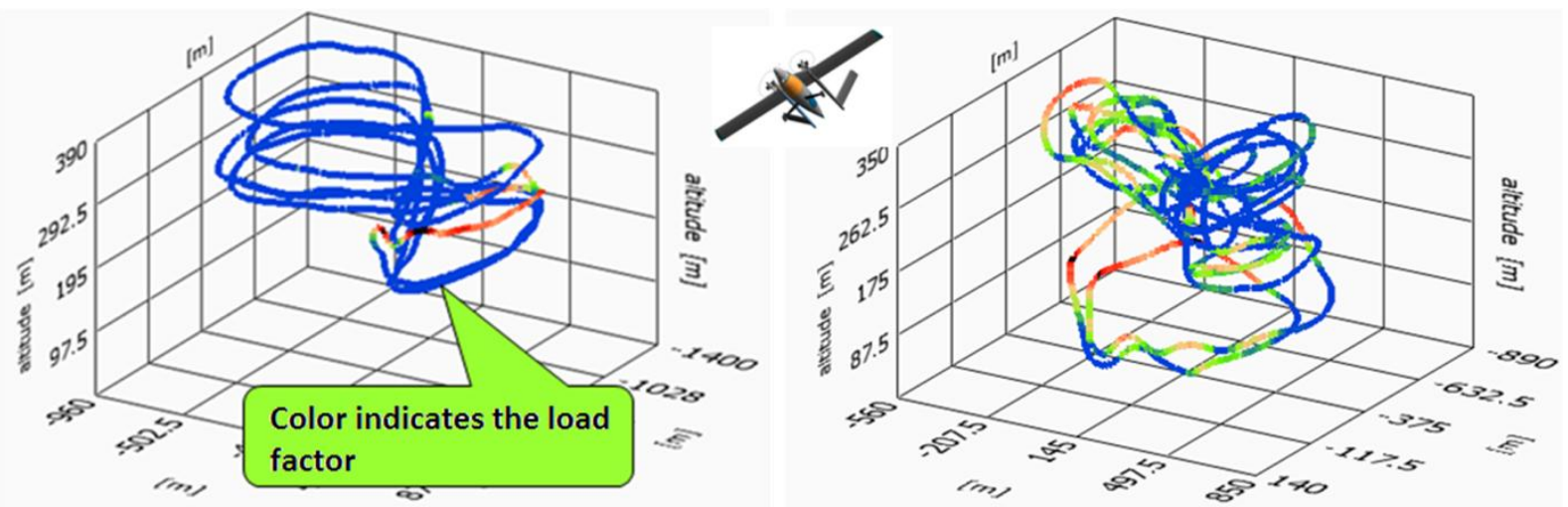

Fig. 11. Differences in the Load Spectra: Auto control mode (left), Manual control mode (right) 

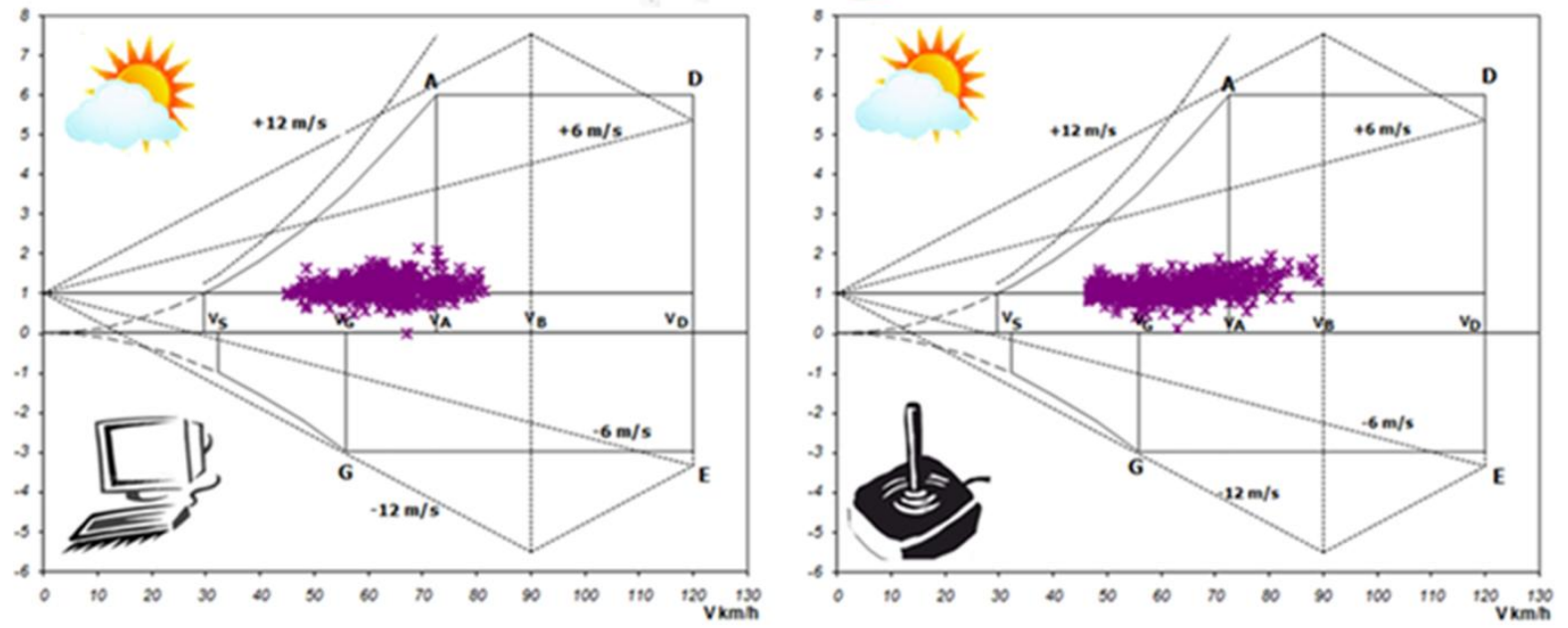

Fig. 12. Loads of a "WorldStar" UAV in different control conditions

Loads caused by air turbulence are essentially independent of the manual control mode. Atmospheric turbulence causes changes in flow and transient increases or decreases in the angles of attack resulting in increases or decreases in aerodynamic forces. The nature of the load is shown in Figure 13.

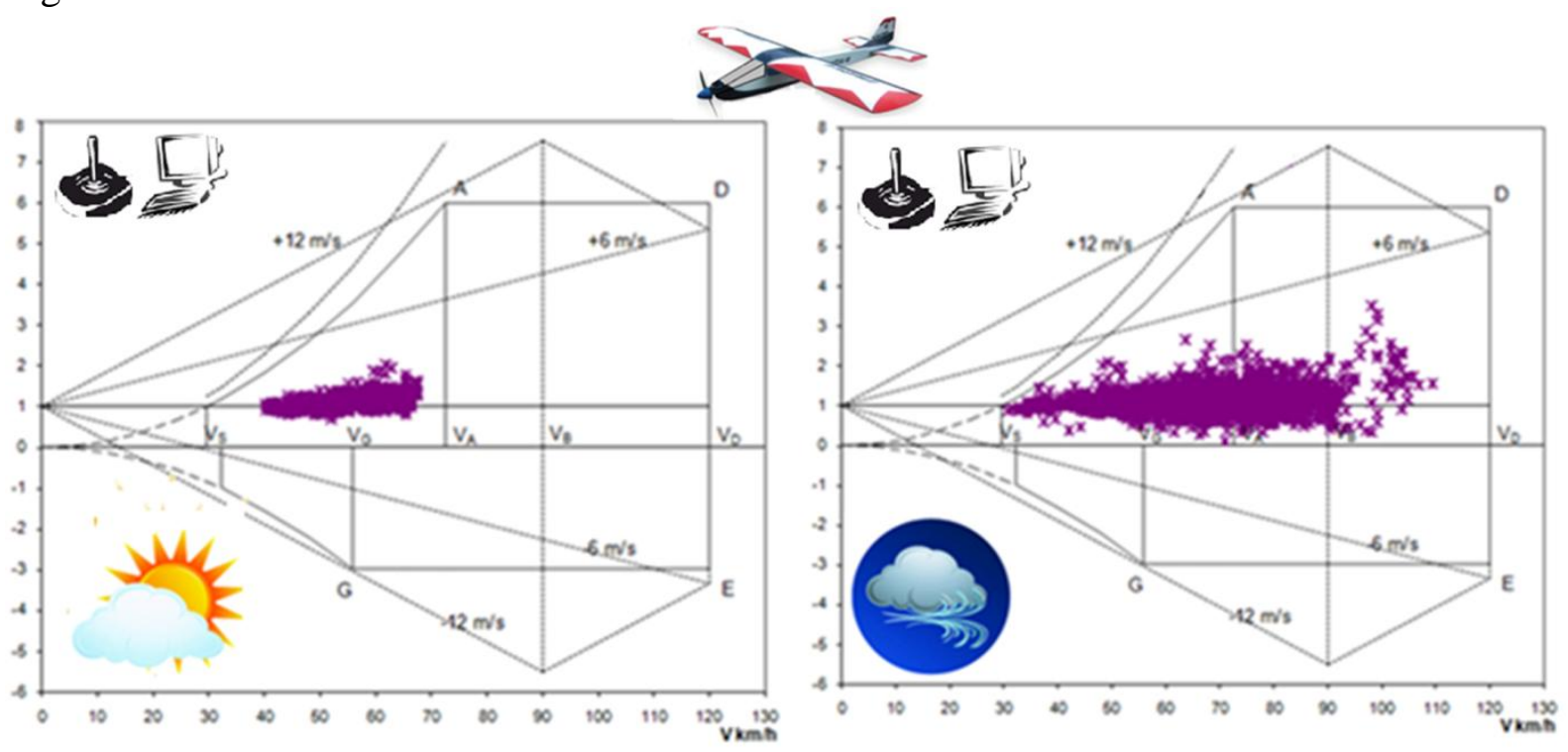

Fig. 13. Loads of a "WorldStar" UAV in different weather conditions

Throughout the largest proportion of its flight, an unmanned aircraft is controlled by an autopilot. However, there are situations in which an operator positioned on the ground has to control the aircraft manually. By examining a typical flight maneuver such as an aerodrome-circle, the incremental difference spectra were prepared (Fig.14). When the airplane is controlled by an autopilot, the quantity of small amplitude load-increments is greater than when it is controlled manually. In the case of manual control, fewer low amplitude load-increments take place, while load-increments of higher variations occur (Fig.14). 


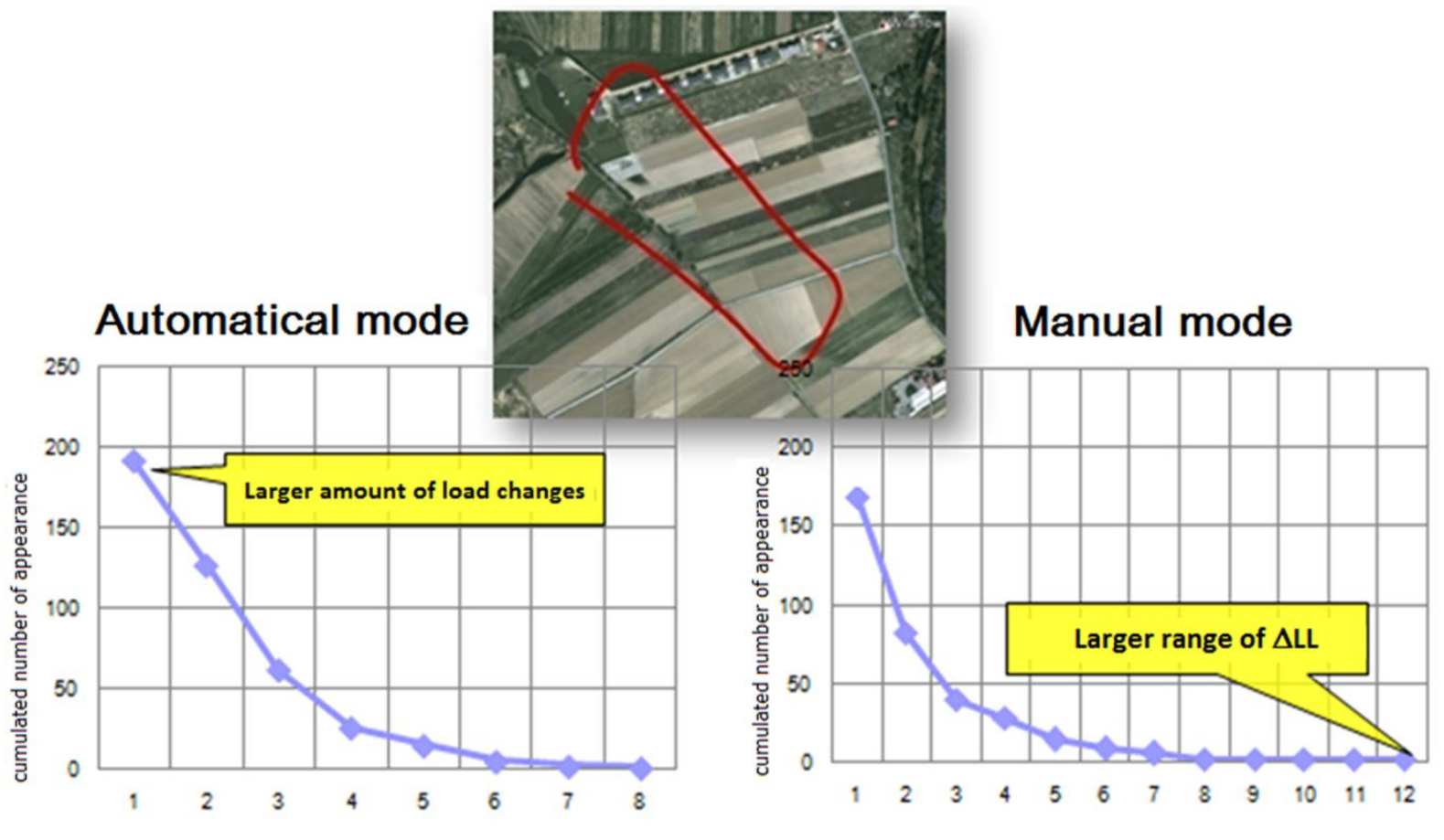

Fig. 14. Load spectrum dependent on automatic and manual mode

It is possible to derive the representative load spectra (RLS) for every type of flight mission (or flight mission part). These are formed as a mean value for the number of occurrences offset vertically by three standard deviations. In the same way the highest value of the load-increment is offset horizontally. Figure 15 shows the RLS for the aerodrome-circles flown by the WorldStar and the fatigue life for aerodrome-circles RLS estimated using the Palmgren-Miner hypothesis.

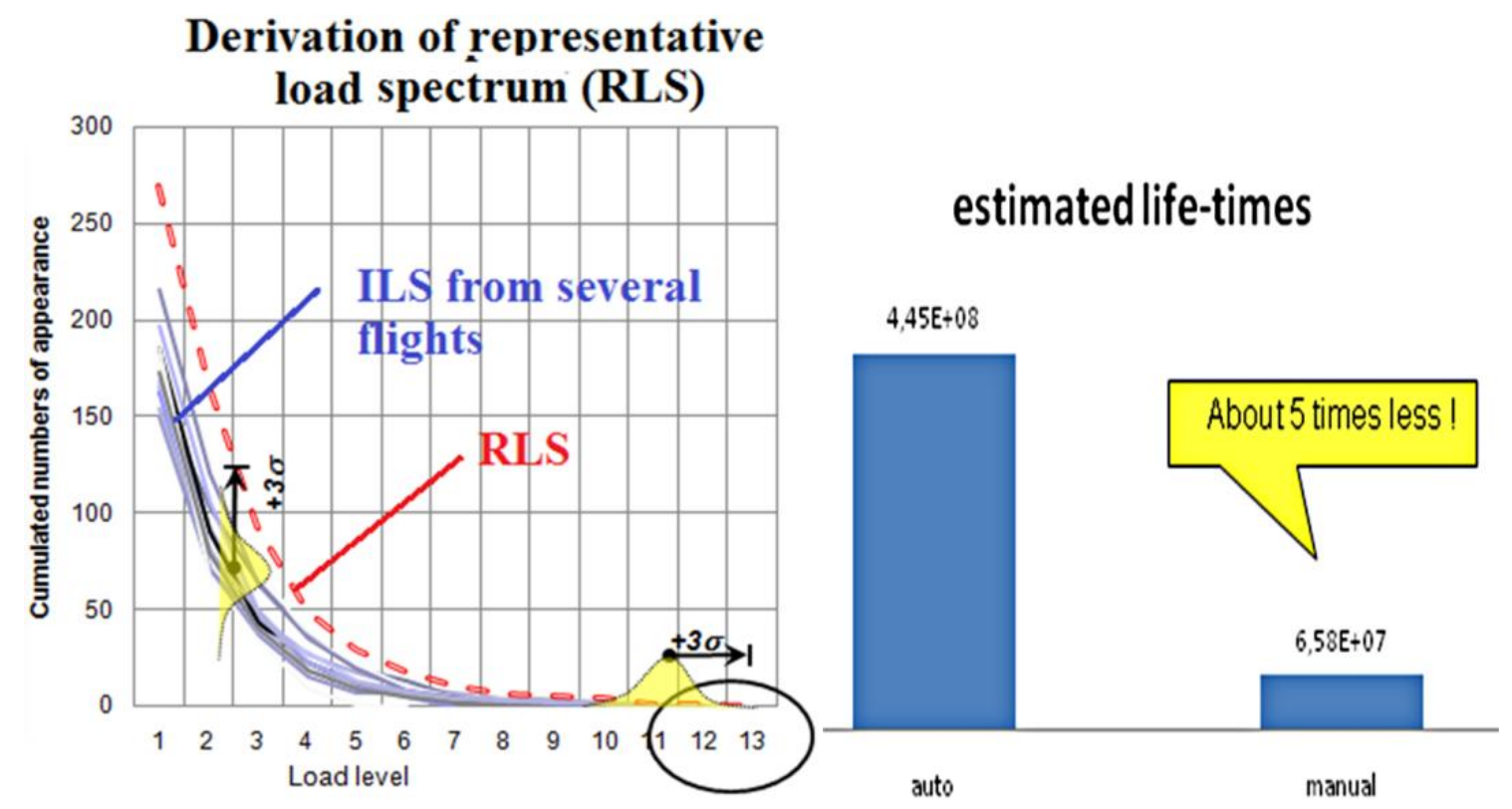

Fig. 15. Representative load spectrum for analyzed flights and estimated life-times with respect to control modes

The authors have developed a method for separating the load spectrum derived from control from that derived from the external, atmospheric turbulence. For this purpose, a simulation model described by a transfer function was built. The input signal was the angle of the elevator 
deflection, whereas the output was the load factor. The transfer function (transmittance) was determined by flight-testing in calm weather. The plane was subjected to sudden change of the elevator angle and then the resulting vertical acceleration was recorded. Transmittance identification was based on a special procedure developed in LabVIEW. Having the transmittance and taking as the input signal the elevator deflection recorded in the flight-log it was possible to simulate the load-signal caused purely by the steering. Subsequently, by comparing the simulated load-signal and the real load-signal recorded in the flight-log it was possible to separate loads caused by the steering from those caused by turbulence (or other sources). Both of the incremental load spectra are juxtaposed for comparison in Figure 16. The visible difference between the measured signal and the simulated one is due to external turbulence and the non-linearity of the model.

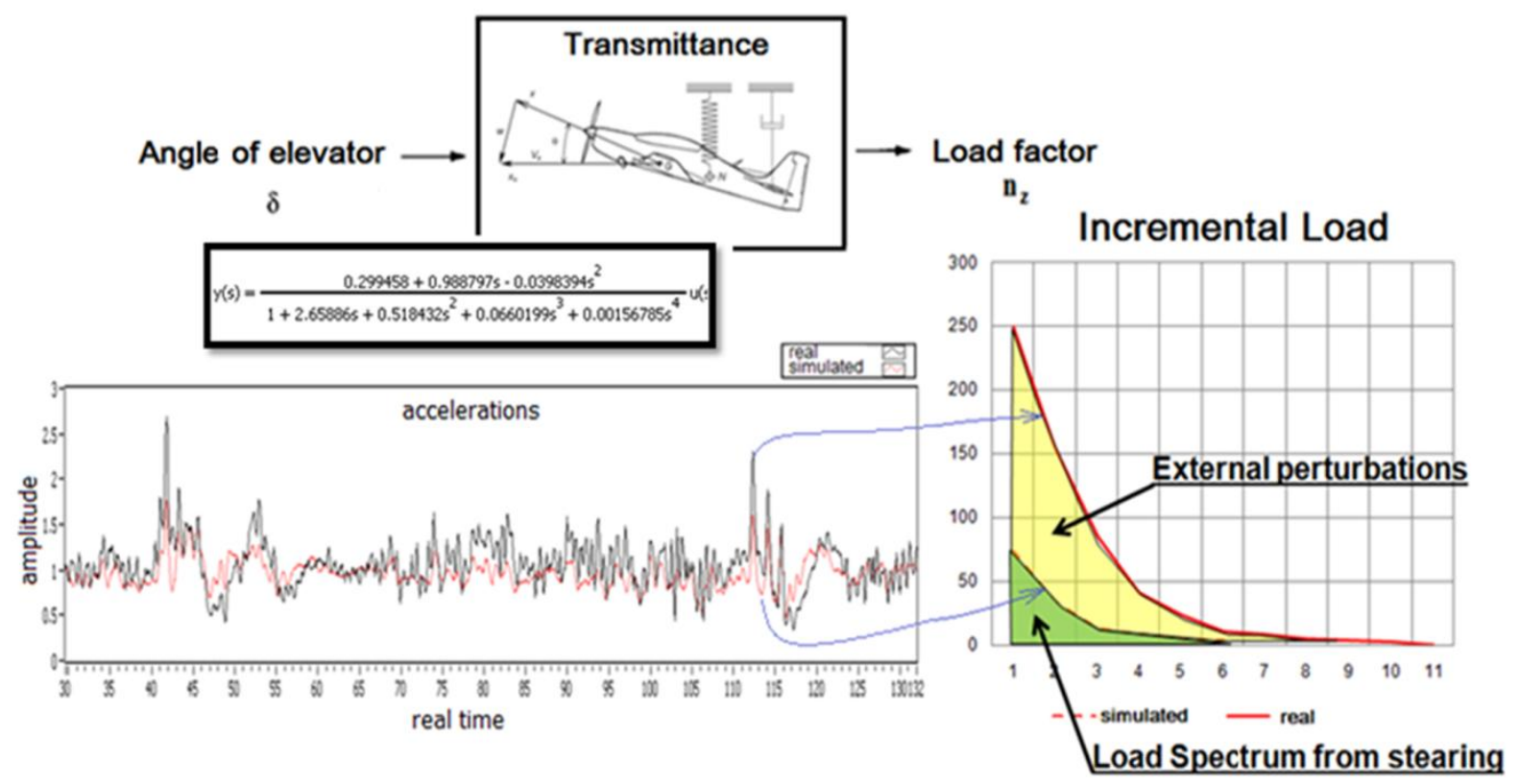

Fig. 16. Comparison of simulated and measured Output signal

\section{RATE OF LOAD VARIATIONS}

Composite materials, which are mainly used for UAV structures, have rheological properties illustrated in Figure 17. Due to these properties, the rate of load changes can have a significant influence on fatigue life. Such calculations would obviously require a fatigue hypothesis that is more sophisticated than the Palmgren-Miner theory, but such investigation would go beyond the scope of the present paper. 


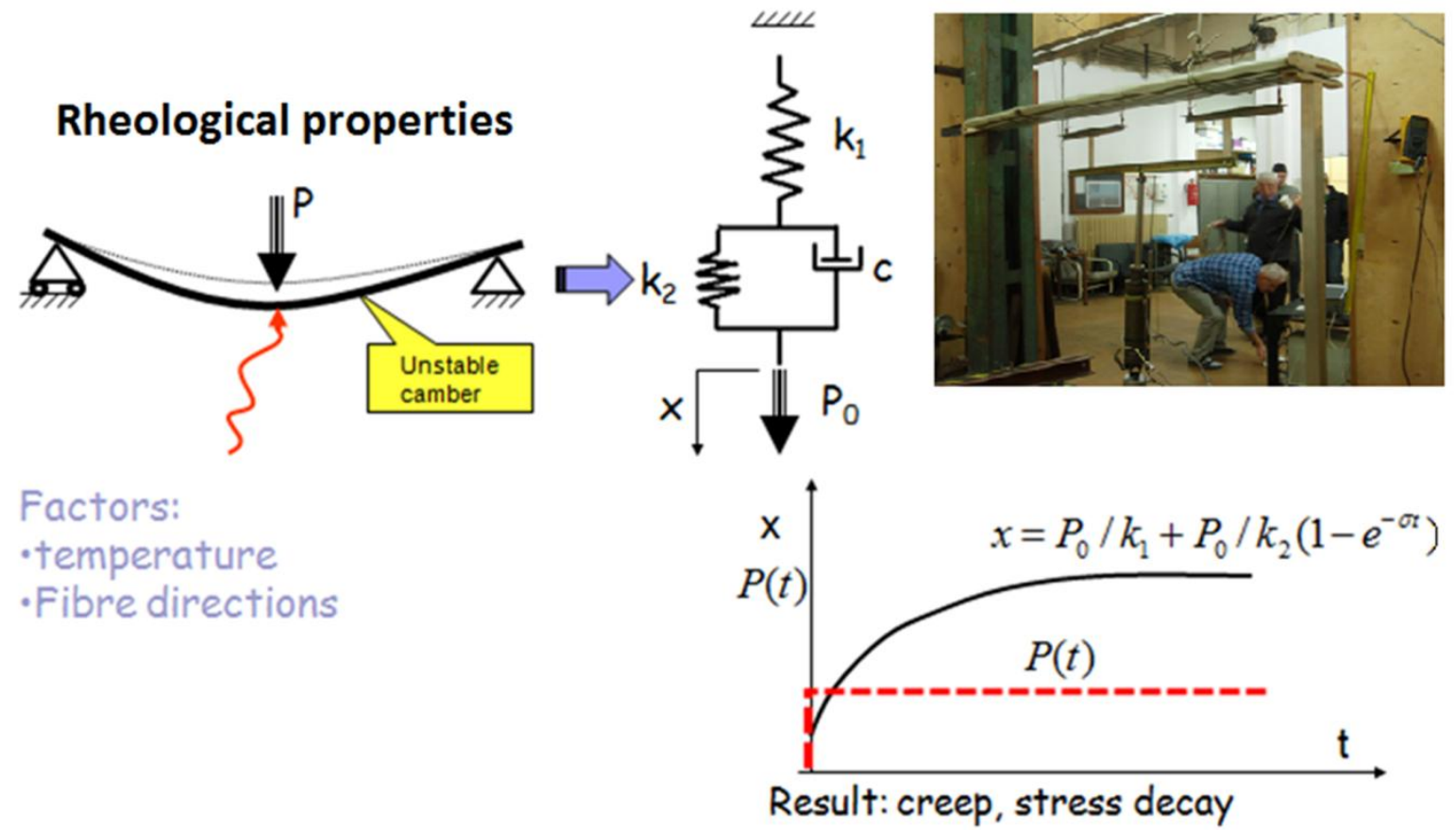

Fig. 17. Composite rheological properties taken into account

Typical algorithms used for deriving load spectra are based only on the load local extremes data-vector, which does not take into consideration the time course. It means that each subsequent change of load occurs in the same time-step as the previous one. It is a very big simplification of the reality (Fig.18).

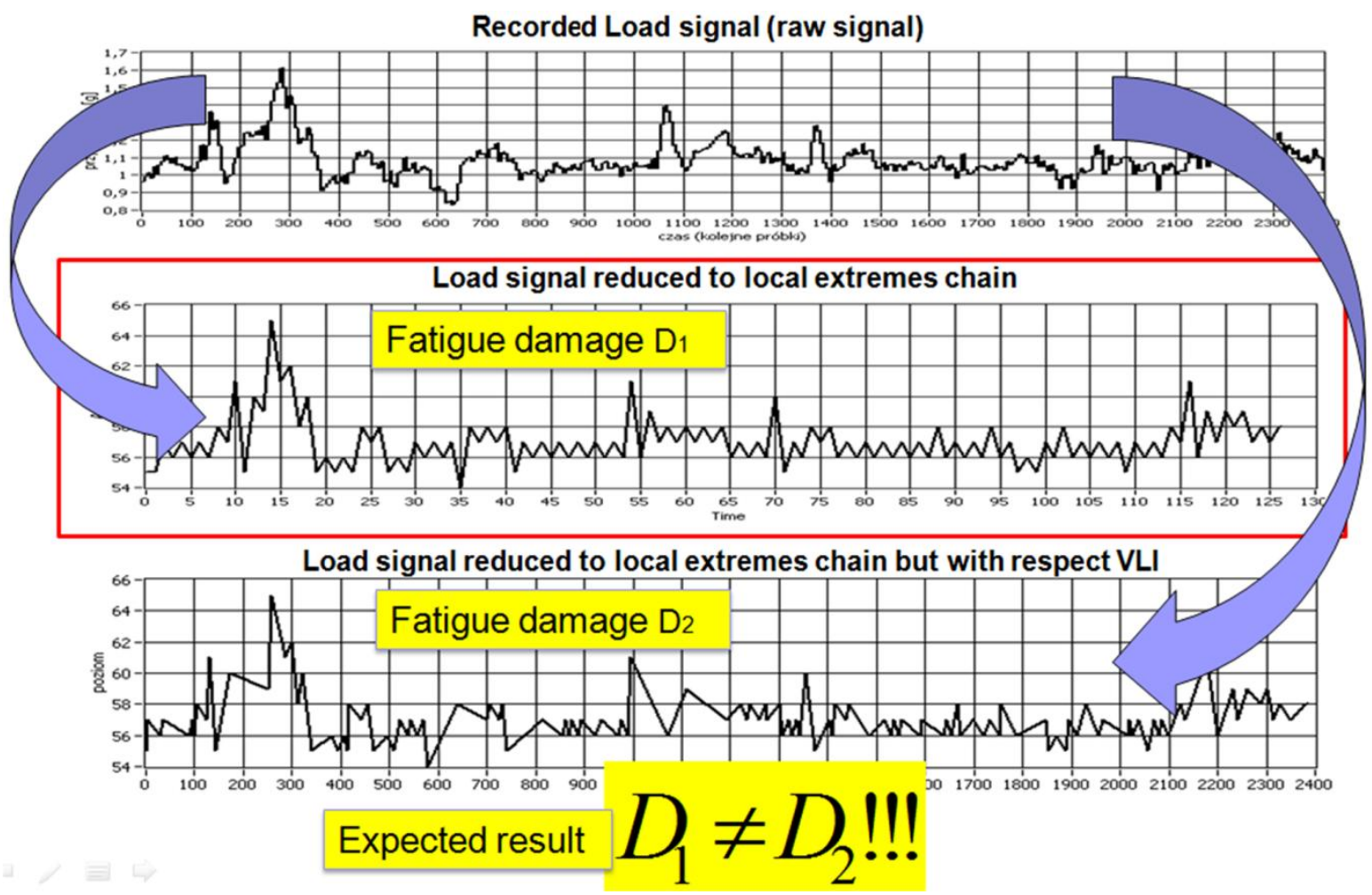

Fig. 18. Local extremes with the rate taken (bottom)/not taken (top) into account

Taking into account the actual rate of load changes it is possible to create arrays that will contain the mean rates of load variations (Fig.19). 


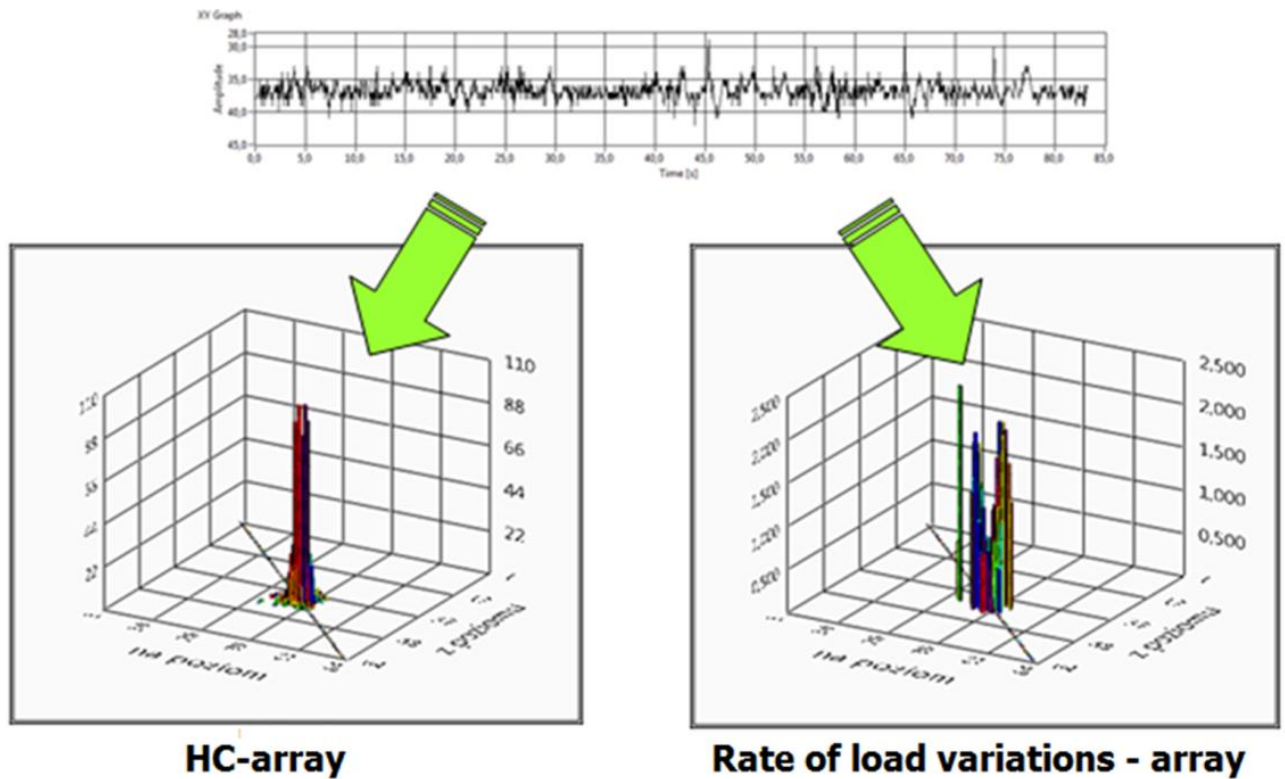

Fig. 19. HC-array and Rate of load variations - array

The algorithm presenting the process of creating such arrays is shown in Fig. 20.

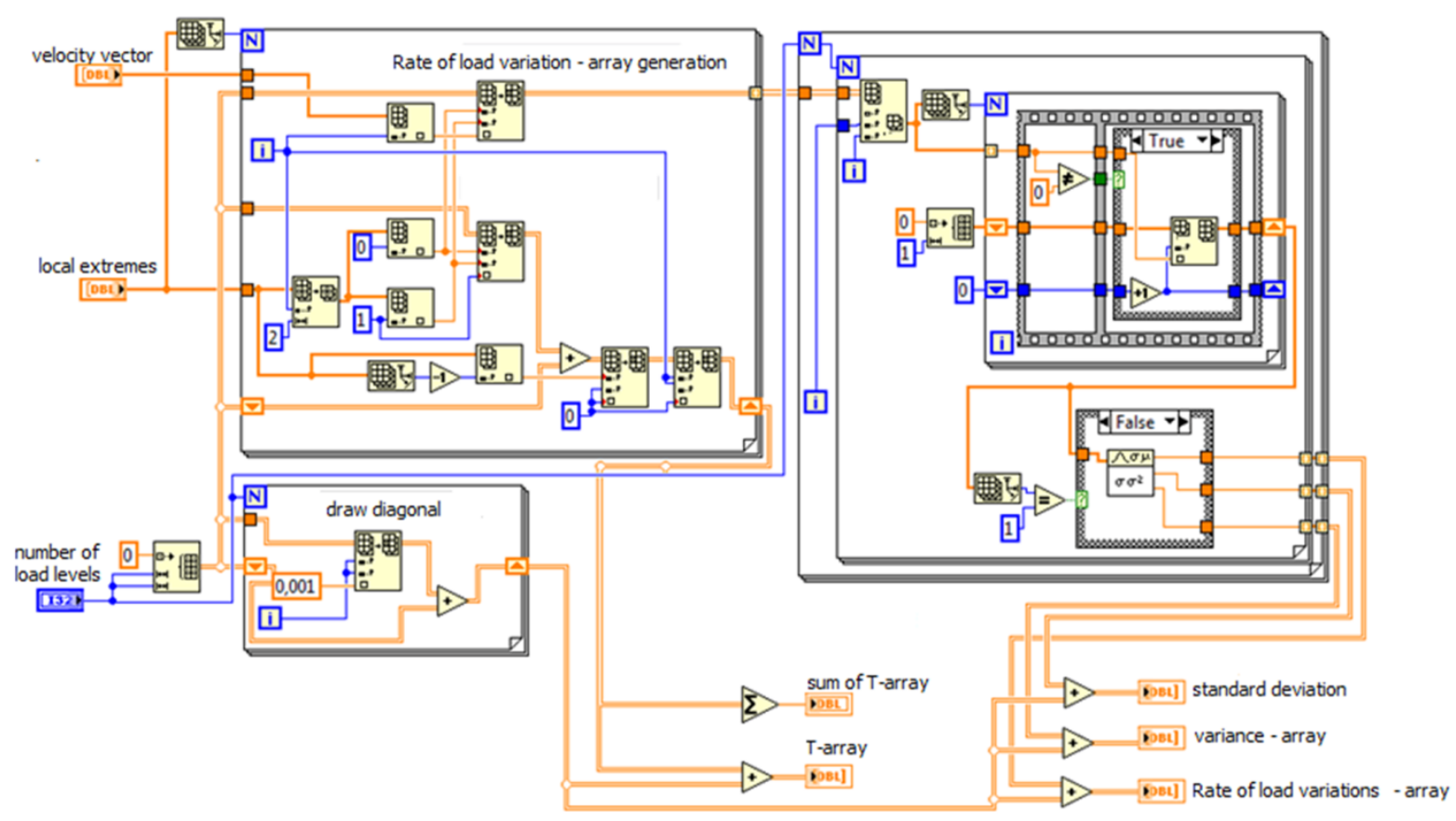

Fig. 20. Derivation of Rate of load variations - array

The results of calculations for different conditions in which the WorldStar aircraft was tested are shown in Figure 21. It was observed that in the case of high turbulence, greater load values corresponded to an increase in the rate of load variations, which might possibly be an important factor in reducing the fatigue life. However, in calm weather conditions, the load increase was accompanied by a decrease in the rate of load variations. 


\section{Rate of load variations}

Turbulent atmosphere
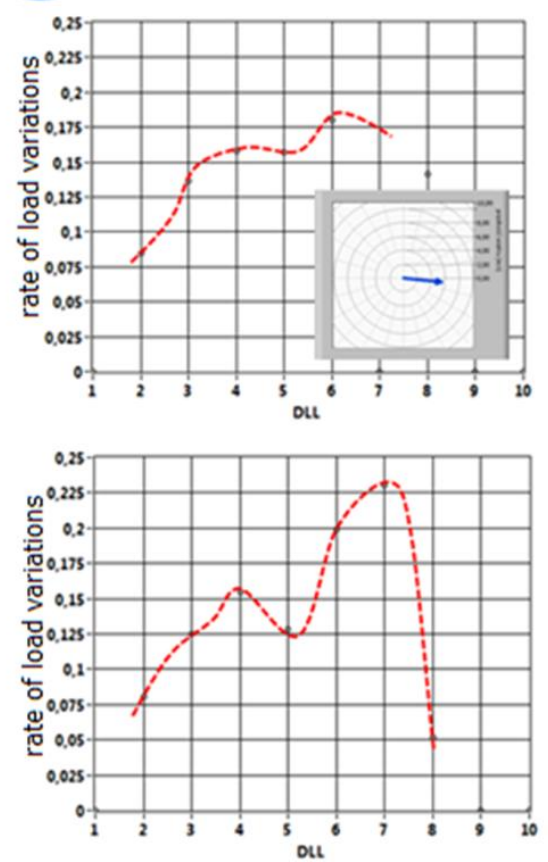

\section{Automatic mode}

\section{Manual mode}

Calm atmosphere
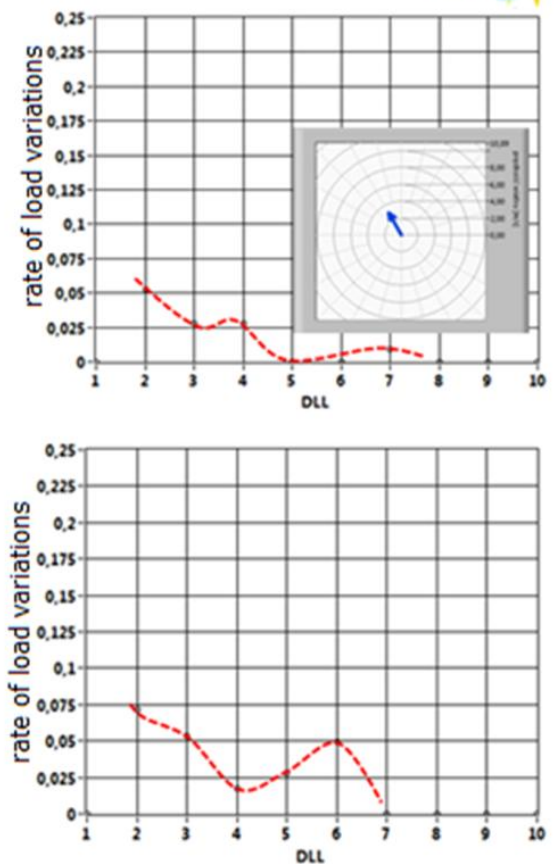

Fig. 21. Rate of load variations recorded during flight tests of the WorldStar Conclusions

\section{SUMMARY}

- Control mode has a big influence on the Load Spectrum.

- The basis for Load Spectra estimations is a statistical analysis of several similar flight missions and consideration of the dispersions of load occurrences number for each load level as well as the dispersion of maximum load increments recorded during flight missions.

- The elaboration process of the model load spectra for fatigue testing of the UAV structure should be based on statistical analysis of possible dispersions of two main parameters: Number of Appearances for each Load Level Increment, and the dispersion of maximum load increments values.

- A modern approach to fatigue testing requires that rheological properties of composites structures are considered.

- Significant differences in the load spectrum between the manual and automatic control modes of the UAV were observed. Those differences have a strong influence on the fatigue life of the UAV structure.

- The determination of UAV load spectra is of utmost importance for the elaboration of airworthiness requirements for this category of aircraft.

- In order to determine the airworthiness requirements regarding fatigue safety of the UAV structure it is necessary to increase the number of analyzed flights - taking into consideration different flight scenarios, different weather and terrain conditions, different pilots, etc. 


\section{REFERENCES}

[1].M.Rodzewicz, D.Głowacki Investigations into load spectrum and strength tests of an UAV structure, The READ 2012 Conference (Research and Education in Aircraft Design) October 2012 Brno

[2].M. Rodzewicz Determination and extrapolation of the glider load spectra. Volume 80 Issue 5 of Aircraft Engineering and Aerospace Technology, pp. 487 - 496

[3].M. Rodzewicz Fatigue evaluation of high-loaded aeronautical composite structures. Aircraft Engineering and Aerospace Technology, Vol 81, No 5, pp 452 - 460

[4]. Rodzewicz M., Czerwiński G., Kubica B. J.: Load spectrum-archiving, analysis and load sequences reproduction (in Polish), Proceedings of XIII Conf. Mechanics in Aviation, PTMTS ML-XIII 2008, vol. 1, pp.43 - 55

[5]. Owczarek W., Rodzewicz M. Investigations into glider chassis load spectrum, Fatigue of Aircraft Structures Monographic Series, Issue 2009, pp 150 - 170. 